\title{
A HOMOTOPY TYPE OF A $p$-GROUP WITH CYCLIC CENTRE
}

\author{
S. B. CONLON \\ (Received 14 April 1983) \\ Communicated by D. E. Taylor
}

\begin{abstract}
Let $G$ be a $p$-group with cyclic centre $\mathscr{Z}(G)=Z$. Then $\mathscr{S}(G)=\left\{Z<H \leqslant G \mid H^{\prime} \cap Z=(1)\right\}$, a poset ordered under inclusion. Then the associated simplicial complex $|\mathscr{S}(G)|$ is homotopic to a bouquet of spheres. A subgroup $E$ of $G$ is called a CES if $C_{G}(E)=Z=\mathscr{Z}(E)$ and if $E / Z$ is elementary. Then $|\mathscr{S}(G)|$ is homotopic to the one-point union of the $|\mathscr{S}(E)|$ for all CES's $E$ in $G$. If $|E / Z|=p^{2 n}$, then $|\mathscr{S}(E)|$ is homotopic to a one-point union of $p^{n^{2}}(n-1)$-spheres.
\end{abstract}

1980 Mathematics subject classification (Amer. Math. Soc.); 20 D 15.

\section{Introduction}

A finite $p$-group $G$ has a faithful irreducible representation $\rho$ over the complex number field $C$ if and only if its centre $\mathscr{Z}(G)=Z$ is cyclic. Indeed by Schur's lemma the restriction of $\rho$ to $Z$ consists of scalar matrices $\lambda I$, where $\lambda$ is a faithful linear representation of $Z$. Every representation of $G$ is monomial and so one can ask if a "transitive" monomial representation with "stabiliser" $H$ restricts to $Z$ to give $\lambda I$. The set of such $H$ gives the poset

$$
\mathscr{S}(G)=\left\{Z<H \leqslant G \mid H^{\prime} \cap Z=(1)\right\},
$$

ordered under inclusion.

$\mathscr{S}(G)$ is unchanged by extension of $Z$ to a larger cyclic group (by central amalgamation or equivalently by the addition of further scalar matrices to the irreducible p-group). Indeed $\mathscr{S}(G)$ is preserved under isoclinism.

1985 Australian Mathematical Society $0263-6115 / 85 \$ A 2.00+0.00$ 
To a poset $X$ is associated a simplicial complex $|X|$ whose vertices are the elements of $X$ and simplices the nonempty chains in $X$. A morphism $f: X \rightarrow Y$ is an order preserving map and induces a simplicial map $|f|:|X| \rightarrow|Y|$ (more details are given in Quillen [2]). For $y \in Y$, we set $f / y=\{x \in X \mid f(x) \leqslant y\}$. We have the following theorem due to Quillen [1]:

(1.1) If $f / y$ is contractible $\forall y \in Y$, then $|f|$ is a homotopy equivalence.

An elementary proof is given by Walker [3].

If instead of $\mathscr{S}(G)$, we consider those "transitive" monomial representations whose "stabilisers" are not contained in the centre $Z$, but which represent $G$ faithfully, we are led to the poset

$$
\mathscr{T}(g)=\left\{H \leqslant G \mid H \nless Z, H^{\prime} \cap Z=(1)\right\} .
$$

However $\mathscr{S}(G)$ and $\mathscr{T}(G)$ are homotopically equivalent. For consider the map $f: \mathscr{T}(G) \rightarrow \mathscr{S}(G), H \mapsto H Z$. If $K \in \mathscr{P}(G)$, then $f / K$ consists of all $H$ in $\mathscr{T}(G)$ with $H \leqslant K$. But any simplex in $|f / K|$ is joined to the vertex $K$. Hence $|f / K|$ is a cone, contractible to $K$, and so by (1.1) |f | is a homotopy equivalence.

Thus we concentrate on $\mathscr{S}(G)$ and show that $|\mathscr{P}(G)|$ is a homotopic to a one-point union of spheres.

A $p$-group $E$ is called an ES if $\mathscr{Z}(E)$ is cyclic and $E / \mathscr{Z}(E)$ is elementary. An ES is almost extras pecial and is extraspecial if its cyclic centre has order $p$. For such an $E$, commutation defines a symplectic form on $E / \mathscr{Z}(E)$ into $\mathbf{F}_{p}$. If $\{E / \mathscr{Z}(E)\}=p^{2 n}, E$ is called an $n$-ES. $E$ is a central amalgamated product of $n$ $1-E S ' s$.

A subgroup $E$ of $G$ is called a CES (centralised ES) if $C_{G}(E)=\mathscr{Z}(E)=Z$ and $E / Z$ is elementary. $E$ is an $n$-CES of $G$ if further $E$ is an $n$-ES.

We will show that $|\mathscr{S}(E)|$ is homotopic to a one-point union of $p^{n^{2}}(n-1)$ spheres when $E$ is an $n$-ES and that $|\mathscr{P}(G)|$ is homotopic to the one-point union of the $|\mathscr{S}(E)|$, and $E$ runs through all CES's in $G$.

\section{2. $|\mathscr{S}(G)|$ as a union of suspensions}

For $K$ such that $Z \leqslant K \leqslant G$, write

$$
\mathscr{A}(K)=\{H \in \mathscr{S}(G) \mid H \leqslant K, H / Z \text { elementary }\} .
$$

If $K=Z$, then $\mathscr{A}(K)$ is empty. In analogy to Proposition 2.1 of Quillen [2], we have:

Propositron 2.1. The inclusion $i: \mathscr{A}(G) \rightarrow \mathscr{P}(G)$ is a homotopy equivalence. 
Proof. Take $K \in \mathscr{S}(G)$. Then

$$
i / K=\mathscr{A}(K)=\{H \mid Z<H \leqslant K, H / Z \text { elementary, } H \text { abelian }\} .
$$

Take $L / Z \leqslant \mathscr{Z}(K / Z)$ with $|L / Z|=p$. If $H \in \mathscr{A}(K)$, then we show that $L H \in \mathscr{A}(K)$. For $L H / Z$ is elementary and so $(L H)^{\prime} \leqslant Z$. But $L H \leqslant K$ and $K^{\prime} \cap Z=(1)$ and so $(L H)^{\prime}=(1)$, i.e. $L H \in \mathscr{O}(K)$. Thus $|i / K|$ is a cone with vertex $L$ and so is contractible. The result now follows from Quillen's Theorem (1.1).

We can now confine our attention to the homotopy type of $\mathscr{A}(G)$. Take $A / Z \leqslant \mathscr{Z}(G / Z)$, with $A / Z$ of order $p$. Let

$$
\mathscr{C}=\left\{H \in \mathscr{A}(G)-\mathscr{A}\left(C_{G}(A)\right)|| H / Z \mid=p\right\} .
$$

Lemma 2.2. $C_{G}(A)$ is maximal in $G$.

Proof. Take $a \in A-Z$. Then $g \mapsto[g, a]$ is a group homomorphism from $G$ onto the subgroup of $Z$ of order $p$. Its kernel is $C_{G}(A)$ which is thus maximal.

Proposition 2.3. $|\mathscr{A}(G)| \simeq \bigvee_{H \in \mathscr{G}} S\left|\mathscr{A}\left(C_{G}(A, H)\right)\right|$ where $\vee$ is the one-point union, $S$ is the (two-point) suspension and $C_{G}(A, H)=C_{G}(\langle A, H\rangle)$.

Proof. For $H \in \mathscr{C}$, set

$$
\mathscr{D}(H)=\{H\} \cup \bigcup_{K \in \mathscr{A}\left(C_{G}(A, H)\right)}\{\langle K, H\rangle\},
$$

a subposet of $\mathscr{A}(G)$. Then

$$
\mathscr{A}(G)=\mathscr{A}\left(C_{G}(A)\right) \cup \bigcup_{H \in \mathscr{C}} \mathscr{D}(H) .
$$

This follows as every $L$ in $\mathscr{A}(G)$ satisfies either

(i) $[L, A]=(1)$, whence $L \in \mathscr{A}\left(C_{G}(A)\right)$ or

(ii) $[L, A]>(1)$, whence $K=C_{G}(A) \cap L$ is maximal in $L$ and so $\exists H \in \mathscr{C}$ with $L=\langle K, H\rangle$ (we allow $L=H$ and $K=Z$ ).

Set $\mathscr{B}=\mathscr{A}\left(C_{G}(A)\right) \dot{\cup} \dot{U}_{H \in \mathscr{E}} \mathscr{D}(H), \dot{U}$ denoting abstract disjoint union. We consider $\mathscr{B}$ as a poset where the order relation within each $\dot{U}$-summand is that of inclusion; if $K \in \mathscr{A}\left(C_{G}(A, H)\right) \subseteq \mathscr{A}\left(C_{G}(A)\right)$, then $K$ in $\dot{\cup}$-summand $\mathscr{A}\left(C_{G}(A)\right)$ is $\leqslant\langle K, H\rangle$ in $\dot{\cup}$-summand $\mathscr{D}(H)$.

It is claimed that the map $f: \mathscr{B} \rightarrow \mathscr{A}(G)$ obtained by removing dots, is a homotopy equivalence. For take $L \in \mathscr{A}(G)$ and look at $f / L$. If $[L, A]=(1)$, then $L \in \mathscr{A}\left(C_{G}(A)\right)$ and $f / L$ is a cone with vertex $L$ lying in the subspace $\left|\mathscr{A}\left(C_{G}(A)\right)\right|$ of $|\mathscr{B}|$. If $[L, A]>(1)$, then either $|L / Z|=p$ and so $L \in \mathscr{C}$ and $f / L=\{L\}$, which is a point and so contractible, or $N=L \cap C_{G}(A)>Z$. In this latter case, 
if $H \in \mathscr{C}$ and $H<L$, then $C_{G}(A, H) \cap L=N$ so that

$$
f / L=\mathscr{A}(N) \cup \bigcup_{\substack{H \in \mathscr{C} \\ H<L}}\left(\{H\} \cup \bigcup_{K \in \mathscr{A}(N)}\{\langle K, H\rangle\}\right) .
$$

In $|\mathscr{B}|$ this is a cone with vertex $N$ and so is contractible. By (1.1) $f$ is then a homotopy equivalence.

It suffices to look at $\mathscr{B}$. The picture of $|\mathscr{B}|$ is first of all a cone $\left|\mathscr{A}\left(C_{G}(A)\right)\right|$ with vertex $A$, together with a separate cone cap with vertex $H$ and section $\left|\mathscr{A}\left(C_{G}(A, H)\right)\right|\left(\subseteq\left|\mathscr{A}\left(C_{G}(A)\right)\right|\right)$ for each $H \in \mathscr{C}$.

We now contract the cone $\left|\mathscr{A}\left(C_{G}(A)\right)\right|$ to its vertex $A$. For each $H \in \mathscr{C}$, the corresponding cone cap of section $\left|\mathscr{A}\left(C_{G}(A, H)\right)\right|$ becomes the suspension $S\left(\left|\mathscr{A}\left(C_{G}(A, H)\right)\right|\right)$ of this section from the two vertices $A$ and $H$. Thus we obtain the one-point union of suspensions

$$
|\mathscr{B}| \simeq \bigvee_{H \in \mathscr{C}} S\left(\left|\mathscr{A}\left(C_{G}(A, H)\right)\right|\right),
$$

with common point $A$, as required.

\section{Case when $G$ is an ES}

Suppose that $G$ is an $n$-ES and so $G$ is the central amalgamated product of $n$ 1-ES's. In applying (2.3), we choose $A / Z \leqslant \mathscr{Z}(G / Z)$ of order $p . C_{G}(A) / Z$ has order $p^{2 n-1}$ and $|\mathscr{C}|=p^{2 n-1}=$ number of points in a projective space of dimension $2 n-1$ over $\mathbf{F}_{p}$ lying outside a hypersurface. Hence there are $p^{2 n-1}$ $\vee$-summands in (2.1). For $H \in \mathscr{C}, C_{G}(A, H) / Z$ has order $p^{2(n-1)}$ and $C_{G}(A, H)$ is an $(n-1)$-ES. By induction on $n$ we can suppose that $\left|\mathscr{A}\left(C_{G}(A, H)\right)\right|$ is homotopic to a one-point union of $p^{2 n-1}(n-2)$-spheres. Each $(n-2)$-sphere suspends to give a $(n-1)$-sphere. Thus the total number of $(n-1)$-spheres in $|\mathscr{A}(G)|$ is $p^{2 n-1} \times p^{(n-1)^{2}}=p^{n^{2}}$ and the induction proceeds. The induction starts when $n=0, G=Z$ and $\mathscr{A}(G)$ is void. $S(\varnothing)$ is the pair of suspending points and so is a 0 -sphere. Summarizing, we have

Proposition 3.1. If $G$ is an $n-E S$, then $|\mathscr{P}(G)|$ is homotopically equivalent to a one-point union of $p^{n^{2}}(n-1)$-spheres.

This structure of $p^{n^{2}}(n-1)$-spheres is the Tits' building for the symplectic group $S p\left(2 n, F_{p}\right)$ acting on $G / Z$ with the symplectic form being given by commutation. The $(n-1)$-dimensional homology group has rank $p^{n^{2}}$ and the induced action of $\operatorname{Sp}\left(2 n, \mathbf{F}_{p}\right)$ on this gives a realisation of the Steinberg character. 


\section{4. $\mathscr{S}(G)$ as a one-point union of spheres}

THEOREM 4.1. Let $G$ be a p-group with cyclic centre $Z$ and set $\mathscr{S}(G)=\{Z<H$ $\left.\leqslant G \mid H^{\prime} \cap Z=(1)\right\}$, ordered under inclusion. Then $|\mathscr{S}(G)|$ is homotopically equivalent to a one-point union of spheres.

Proof. By (2.1), $|\mathscr{S}(G)| \simeq|\mathscr{A}(G)|$ and by (2.3) we have

$$
|\mathscr{A}(G)| \simeq \bigvee_{H \in \mathscr{C}} S\left(\left|\mathscr{A}\left(C_{G}(A, H)\right)\right|\right)
$$

where $\mathscr{C}=\left\{H \in \mathscr{A}(G)-\mathscr{A}\left(C_{G}(A)\right)|| H / Z \mid=p\right\}$. We apply this same result (4.2) to each $\vee$-summand $\mathscr{A}\left(C_{G}(A, H)\right)$ in turn and so on. We thus obtain $\vee$-sums over sequences $\left(A, H ; A_{1}, H_{1} ; \ldots\right)$ and we look at how these sequences terminate. For a particular choice of $A, H$ we look at $S\left(\left|\mathscr{A}\left(C_{G}(A, H)\right)\right|\right)$.

(i) Case $\mathscr{Z}\left(C_{G}(A, H)\right)>Z$. Take $B \leqslant \mathscr{Z}\left(C_{G}(A, H)\right)$ with $|B / Z|=p$. Then $\left|\mathscr{A}\left(C_{G}(A, H)\right)\right|$ is contractible to the vertex $B$. As $S$ (point) $\simeq$ point, such an ending gives no contribution to final one-point union.

(ii) Case $G_{1}=C_{G}(A, H)>Z$ and $\mathscr{Z}\left(G_{1}\right)=Z$. Choose $A_{1} / Z \leqslant \mathscr{Z}\left(G_{1} / Z\right)$ with $\left|A_{1} / Z\right|=p$. If $\mathscr{A}\left(G_{1}\right) \nsubseteq \mathscr{A}\left(C_{G_{1}}\left(A_{1}\right)\right)$, then a choice of $H_{1}$ is possible and sequence proceeds. If however we have $\mathscr{A}\left(G_{1}\right) \subseteq \mathscr{A}\left(C_{G_{1}}\left(A_{1}\right)\right)$, then every element of $\mathscr{A}\left(G_{1}\right)$ commutes with $A_{1}$ and $\left|\mathscr{A}\left(G_{1}\right)\right|$ is homotopic to a cone with vertex $A_{1}$ and so is contractible to a point. As in (i), this gives no contribution to the final one-point union.

(iii) Case $C_{G}(A, H)=Z$. Thus $\mathscr{A}\left(C_{G}(A, H)\right)$ is void and $S(\varnothing)$ is the two-point 0 -sphere. Continuing suspensions give higher dimensional spheres (as in Section 3).

Hence nontrivial contributions to $|\mathscr{A}(G)|$ come from sequences $A_{1}, H_{1} ; \ldots ; A_{n}, H_{n}$, where, if we set $E=\left\langle A_{1}, H_{1}, \ldots, A_{n}, H_{n}, Z\right\rangle$, then $C_{G}(E)=$ $Z$ and so $E$ is an $n$-CES for some $n$. Each such $\vee$-summand is homotopic to the $n$-fold suspension $S^{n}(\varnothing)$ which is a $(n-1)$-sphere and so $|\mathscr{A}(G)|$ is a one-point union of spheres, as required.

\section{Critical roles of the CES's}

(5.1) An $(n-1)$-spherical $\vee$-summand in (4.1) corresponds to a sequence $A_{1}, H_{1} ; \ldots ; A_{n}, H_{n}$. These subgroups together with $Z$ generate an $n$-CES $E$ of $G$. 
We now collect together summands in (4.1) according to the CES that they generate.

LEMMA 5.2. If $A / Z$ lying in $\mathscr{Z}(G / Z)$ has exponent $p$ and if $E$ is $C E S$ in $G$, then $A \leqslant E$.

Proof. As an elementary group is generated by its subgroups of order $p$, it is sufficient to show the result when $A / Z$ has order $p$.

Suppose $E$ is an $n$-CES and so $E / Z$ has order $p^{2 n}$. If $A \nless E$, then $A E / Z$ is elementary of order $p^{2 n+1}$. Commutation defines a symplectic form on $A E / Z$ into $F_{p}$ and as this has odd dimension over $F_{p}$ it has a singular subspace $Y / Z$. Then $Z<Y \leqslant C_{G}(E)$, contrary to the fact that $E$ is a CES. Hence $A \leqslant E$, as required.

THEOREM 5.3. Let $G$ be a p-group with cyclic centre $Z$. Then $|\mathscr{P}(G)|$ is homotopic to a one-point union

$$
|\mathscr{S}(G)| \simeq \vee|\mathscr{P}(E)|
$$

where $E$ runs through the CES's of $G$. If $E$ is an n-CES, then $|\mathscr{P}(E)|$ is homotopic to a one-point union of $p^{n^{2}}(n-1)$-spheres.

Proof. Let $E$ be an $n$-CES of $G$. As $|\mathscr{S}(G)|$ and $|\mathscr{S}(E)|$ are one-point unions of spheres, it is sufficient to see that there are sufficient (i.e. $\left.p^{n^{2}}\right) \vee$-summands in (4.1), indexed by sequences $\left(A_{1}, H_{1} ; \ldots ; A_{n}, H_{n}\right)$, such that $E=\left\langle A_{1}, \ldots, H_{n}, Z\right\rangle$.

Take $r$ with $0<r<n$ and write $M_{r}=\left\langle A_{1}, \ldots, H_{r}, Z\right\rangle$ and $N_{r}=$ $\left\langle A_{r+1}, \ldots, H_{n}, Z\right\rangle$. Then $E$ is the central amalgamated product of the ES's $M$, and $N_{r}$. At the $(r+1)$ st stage of analysing and forming the summands in (4.1) we have to look at $C_{G}\left(A_{1}, H_{1}, \ldots, A_{r}, H_{r}\right)=C_{G}\left(M_{r}\right)$ and choose $A_{r+1} / Z$ in $\mathscr{Z}\left(C_{G}\left(M_{r}\right)\right)$ of order $p$. Now $N_{r}$ is a CES in $C_{G}\left(M_{r}\right)$ and so by (5.2) $A_{r+1} \leqslant N_{r}$. A union is then taken over all $H_{r+1} / Z$ of order $p$ in $C_{G}\left(M_{r}\right)$ with $\left[A_{r+1}, H_{r+1}\right]>(1)$. Considering only those $H_{r+1} / Z$ which lie in a given $n$-CES $E$, we see that their number is independent of the rest of $G$ as is the same as if $E$ were considered in isolation. Hence that part of the one-point union $|\mathscr{S}(G)|$ in 4.1 coming from all sequences $A_{1}, \ldots, H_{n}, Z$ which generate $E$ is homotopic to $|\mathscr{S}(E)|$. This completes the proof. 


\section{References}

[1] D. Quillen, 'Higher algebraic $K$-theory I', pp. 85-147, (Lecture Notes in Mathematics, 341, Springer-Verlag, Berlin, 1973).

[2] D. Quillen, 'Homotopy properties of the poset of nontrivial $p$-subgroups of a group', $A d v$. in Math. 28 (1978), 101-128.

[3] James J. Walker, 'Homotopy type and Euler characteristic of partially ordered sets', European J. Combin. 2 (1981), 373-384.

Department of Pure Mathematics

University of Sydney

Sydney, N.S.W. 2006

Australia 